\title{
Minimum Degree Reordering Based Graph Partitioning Method for Distributed Fault Section Estimation System in Power Networks
}

\author{
Tianshu Bi, St.M. IEEE Yixin Ni, S.M. IEEE Felix F. Wu, Fellow IEEE Qixun Yang* \\ Dept. of Electrical and Electronic Engineering \\ The University of Hong Kong \\ Hong Kong SAR \\ *Dept. of Electrical Engineering \\ North China Electric Power University \\ Beijing, China
}

\begin{abstract}
In order to make fault section estimation (FSE) in largescale power networks use distributed artificial intelligence approach, we have to develop an efficient way to partition the large-scale power network into desired number of connected subnetworks such that each sub-network should have quasi-balanced working burden in performing FSE. In this paper, an efficient minimum degree reordering based graph partitioning method is suggested for the partitioning task. The method consists of two basic steps: partitioning the power network into connected, quasibalanced and frontier minimized sub-networks based on minimum degree reordering and minimizing the number of the frontier nodes of the sub-networks through iterations so as to reduce the interaction of FSE in adjacent sub-networks. The partitioning procedure and characteristic analysis is presented. The method has been implemented with sparse storage technique and tested in the IEEE 14-bus, 30-bus and 118-bus systems respectively. Computer simulation results show that the proposed multiple-way graph partitioning method is suitable for FSE in large-scale power networks and is compared favorably with other graph partitioning methods suggestcd in refcrences.
\end{abstract}

Keywords graph partitioning, fault section estimation, large-scale power networks

\section{INTRODUCTION}

Fault section estimation aims at identifying the faulted element(s) in power networks by using the information of the current status of protective relays and circuit breakers. As the first step to system restoration, FSE is of great importance in enhancing service reliability and reducing power supply interruption. FSE should be implemented quickly and accurately in order to isolate the faulty elements from the rest of the system and to take proper countermeasures to recover normal power supply. It is clear that on-line automatic FSE is significant and crucial to the restorative operations.

Although many techniques have been utilized to solve the problem, such as expert-system-based ${ }^{[1-2]}$, fuzzy-setbased [3], artificial-neural-network-based [4-5], stochasticoptimization-based ${ }^{[6]}$ and logic-based ${ }^{[7]}$ approaches, fault section estimation in large-scale power networks still remains as a difficulty and unsolved issue because of the large amount of information to be dealt with and the FSE speed and accuracy required. The FSE is even more difficult in cases with failure operations of relays and circuit brcakers, or multiple faults at the same time.

Since faults and signals from relevant relays and circuit breakers are basically local phenomena, based on the idea of 'divide and conquer', we suggest to use distributed artificial intelligence system for fault section estimation in large-scale power networks, which is more reasonable and more efficient than traditional centralized FSE system. It is clear that in order to implement the task of distributed fault section estimation system well a good power network partitioning method is essential and crucial.

If we denote the buses and transmission lines in power networks as vertices and edges of a graph, the power network partitioning problem can be easily transformed into a corresponding graph partitioning problem. That is to partition the graph vertices into desired number of connected and quasi-balanced subsets according to the weights of the vertex under the constraint that the number of frontier nodes crossing the different subsets is minimized.

This graph partitioning problem is $N P$-hard ${ }^{[8]}$. Efficient heuristic algorithms have been investigated for its solution, such as Kernighan-Lin heuristics ${ }^{[9-10]}$, stochastic optimization based methods ${ }^{[11-12]}$ and graph partitioning methods for parallel computation ${ }^{[13-14]}$ etc. The first two methods realize the optimal partition by aggressive and stochastic searches respectively, which partition the graph into balanced subsets under the constraint that the number of edges crossing the subsets is minimized; while the third method puts more emphasis on reducing the nonzero elements in generating balanced partition. Therefore, the common demerit of these methods is that none of them consider the special requirement of the FSE that any obtained sub-network must be connected. It is not easy to modify these methods to adapt this requirement.

In this paper an efficient minimum degree reordering ${ }^{[15-}$ 16] based graph partitioning method for distributed fault section estimation system in large-scale power networks is proposed. The partitioning procedure and characteristic analysis is presented. The method has been implemented with sparse storage technique and tested in the IEEE 14bus, 30-bus and 118-bus power systems. For comparison purpose the graph partitioning method based on depth-firstsearch algorithm ${ }^{[17]}$ has been realized and tested as well. Computer test results demonstrate that the proposed multiple-way graph partitioning approach is suitable for distributed fault section estimation system in large-scale power networks and has better quality as compared with the graph partitioning method based on depth-first-search algorithm.

\section{PROPOSED GRAPH PAKTITIONING METHOD}

The proposed multi-way graph partitioning method consists of two basic steps: realizing an initial partition by the multi-way graph partitioning algorithm based on weighted minimum degree reordering and further minimizing the number of the frontier nodes through iterations so as to reduce the interaction of FSE in adjacent sub-networks. The two steps with their formulations and theoretical basis are presented in detail below. 
A. Multi-way graph partitioning algorithm based on weighted MD reordering

Suppose $G$ is a labeled undirected graph with $n$ vertices. Minimum degree (MD) reordering ${ }^{[15]}$ can be best described in terms of elimination graphs. The number of edges incident on the node $x$ is called degree of node $x$. After eliminating the node $x$ from the graph $G$, the corresponding elimination graph can be obtained by deleting the node $x$ and its incident edges and then adding edges between any pair of nodes $x_{j}$ and $x_{k}$ for which $\left(x, x_{j}\right)$ and $\left(x, x_{k}\right)$ belong to the graph $G$ but $\left(x_{j}, x_{k}\right)$ does not. The elimination process can thus be modeled by a sequence of graphs, each having one less node than the previous graph, until only one node remains. At each step of the elimination process, MD algorithm selects as the next node to be eliminated a node of minimum degree in the current elimination graph.

A part of the IEEE 118-bus system is taken as the illustrative example (Fig. 1), in which each vertex is denoted by the original subscript and corresponding $\mathrm{MD}$ reordering directly. The dotted lines represent the added edges in the elimination process. Using MD algorithm, we could get the reordering is 87868483888590918982 94929677.

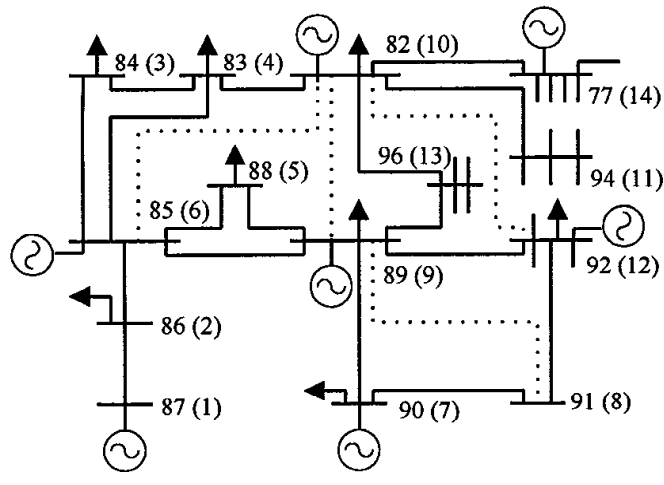

Fig.1 The illustrative power network for $\mathrm{MD}$ algorithm

In addition, in order to get the balanced partition, we assign each vertex an integer, called weight, to form the weighted MD reordering. The investigation shows that the calculation burden of a sub-graph is mainly determined by the total number of possible fault elements in the network, hence the weight of a vertex is defined as the number of its incident possible fault elements (bus, lines and transformers).

Suppose $Y_{n}$ is the bus admittance matrix of the given power network in descent order of the reordering label. For node $x_{t}$, we use the number of the nonzero elements in row $i$ of the upper triangular matrix (including the diagonal element) of $Y_{n}$ as its node weight and denote it as nodewt $\left(x_{1}\right)$. With the weights, the previously formed MD reordering becomes a weighted $\mathrm{MD}$ reordering. Then the weighted $\mathrm{MD}$ reordering of the illustrative power network is 87 (2) 86 (2) 84 (3) 83 (3) 88 (3) 85 (2) 90 (3) 91 (2) 89 (2) 82 (3) 94 (4) 92 (2) 96 (2) 77 (3).

For graph $G$ with $n$ vertices, let $x_{1}, x_{2}, \ldots, x_{n}$ be the obtained weighted MD reordering, Weights denote the total weights of $n$ vertices and $n_{g}$ be the desired number of subgraphs. It can be observe, along the MD reordering, each node will either merge some preceding disjoint subsets into a connected cluster, or exist as a new disjoint subset. If the weight of the emerging connected component is most approaching Weights $/ n_{g}$, then nodes in this component make up the first desired sub-graph $C_{l}$. Delete $C_{I}$ from the reordering and continue the above process iteratively until all the nodes in graph $G$ have been explored. The completed multi-way graph partitioning algorithm based on weighted MD reordering is described in Algorithm 1.

Algorithm 1. Proposed multi-way graph partitioning algorithm

Step 1: Denote the MD reordering of $G$ by $S$, the node sets of the subgraphs by $C_{l}$, where $i=1,2, \ldots, n_{g}$, and the preceding disjoint clusters in the partition process by $D_{j}, j=1,2, \ldots, k$, where $k$ is a variable. Initially, set $S=\left\{x_{i}, x_{2}, \ldots, x_{n}\right\}, C_{i}=\varnothing$, and $D j=\varnothing$;

Step 2: Set the circulation pointer $p=1$. The first node $x_{l}$ in the reordering composes $D_{l}$, that is, $D_{l}=\left\{x_{l}\right\}$;

Step 3: $p=p+1$;

Step 4: For node $x_{p}$ in the ordering, find all the preceding disjoint connected clusters $\left\{D_{j}\right\}$ which are incident with $x_{p}$, that is $\left\{D_{j} \mid\right.$ $\left.\exists x_{i} \in D_{j}, x_{i} x_{p} \in G\right\}$, and then these clusters and node $x_{p}$ make up a new connected cluster $D_{p}=\left\{D_{f}\right\} \cup x_{p}$. Sort the clusters $\left\{D_{j}\right\}$ in descending order according to their cluster weights $w t\left(D_{j}\right)$. Then test these clusters $D_{J}$ one by one by this sequence, if $D_{j}$ satisfies:

$\mid w t\left(D_{p}\right)-$ Weights $/ n_{\mathrm{g}}|>| w t\left(D_{j}\right)-$ Weights $/ n_{\mathrm{g}} \mid$

This means $D$ is most approaching Weights $/ n_{g}$, then assign this connected cluster $D_{j}$ as a sub-graph $C_{i}=D_{j}$ and $i=i+1$. At the same time delete all the nodes in $D_{j}$ from the cluster $D_{p}$ and the ordering $S$ respectively. If all the preceding disjoint clusters $\left\{D_{j}\right\}$ associated with $x_{p}$ couldn't satisfy (1), replace all these associated clusters $\left\{D_{\}}\right\}$by $D_{p}$;

Step 5: If $p$ equals $n$, then $S=\varnothing$ means all the nodes of $G$ have been explored systematically, and so the algorithm terminates, else repeat Step 3 to Step 5.

For the weighted MD reordering 87 (2) 86 (2) 84 (3) 83 (3) 88 (3) 85 (2) 90 (3) 91 (2) 89 (2) 82 (3) 94 (4) in Fig. 1, suppose $n_{\mathrm{g}}=2$ and Weights $=29$. Then node 85 merges three disjoint subsets: $\{87,86\},\{84,83\}$ and $\{88\}$ into one connected component, while the following node 90 makes up a new disjoint subset $\{90\}$. The weight of this new connected component is 15 , which is most approaching

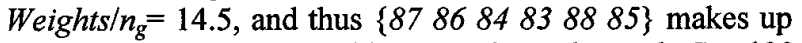
$C_{1}$. The same way we could get another sub-graph $C_{2}=\{90$ $91898294\}$.

It is obvious that the weighted MD reordering based multi-way graph partitioning algorithm guarantees that the calculation burdens are distributed evenly because all the partitions satisfy (1), but the perceptive reader may question the proposed algorithm in terms of the connectivity and minimization of the frontiers of the subgraphs. Aiming at these problems, further characteristic analysis is conducted.

It can be observed that the sub-graph $C_{I}$ could always be obtained successfully by Algorithm 1 . However, if the rest part of the graph $G$ becomes disconnected, Algorithm 1 can not finish the partitioning task as we expected. Therefore the key point of the connectivity of Algorithm 1 is that the rest part of the graph $G$ must be connected after all the obtained sub-graphs have been eliminated. We'll give proof to this point.

The proof needs the help of a tree structure called the elimination tree ${ }^{[16]}$, whose related concepts and lemma are introduced first. Assume $Y_{n}$ is the large sparse symmetric positive definite matrix of the graph $G$, and $L$ is its cholesky factor. If $x_{1}, x_{2}, \ldots, x_{n}$ is the ordering of the matrix $Y_{n}$, the elimination tree of $Y_{n}$ is defined to be the tree with the same set of $n$ nodes, and $x_{t}$ is the parent node of $x_{j}$ if and only if: 


$$
i=\min \left\{k \mid l_{k j} \neq 0\right\}
$$

The structure as defined is a tree with $n$ nodes rooted at the node $x_{n}$, which is denoted by $T\left[x_{n}\right]$. The same way, for any node $x_{j}$ in the elimination tree, the subtree rooted at $x_{j}$ is denoted by $T\left[x_{j}\right]$. If the elimination tree is produced on the basis of the MD reordering, it has the following desired property.

Lemma 1. [16] For each node $x_{j}$ in the elimination tree produced by MD reordering of $G$, the sub-graph of $G$ consisting of nodes in $T\left[x_{j}\right]$ is connected and is given by the connected component $C_{j}$ in the MD reordering $\left\{x_{1}, \ldots, x_{j}\right\}$ containing $x_{j}$.

Based on the concepts and Lemma 1, we further present and prove Lemma 2 and Theorem 3, which provide a sound theoretical basis for connectivity of the proposed algorithm.

Lemma 2. For each node $x_{j}$ in the elimination tree produced by MD reordering of $G$, the sub-graph of $G$ consisting of nodes, $\left\{x_{i} \mid x_{i} \notin T\left[x_{j}\right]\right\}$, not in $T\left[x_{j}\right]$ is connected too.

Proof. For any node $x_{j}$ in the elimination tree, the ordering obtained from an elimination tree rotation ${ }^{[16]}$ (actually a composite of basic rotations) at $x_{j}$ will maintain the relative ordering of the nodes that are not ancestors of $x_{j}$. But the ancestor nodes of $x_{j}$ will be reordered such that the nodes in $\operatorname{Adj}\left(T\left[x_{j}\right]\right) \cup\left\{x_{j}\right\}$ are numbered last. Since the set $\operatorname{Adj}\left(T\left[x_{j}\right]\right)$ $\cup\left\{x_{j}\right\}$ is a clique in the elimination graph, they can be renumbered among themselves in any order so long as they are labeled last. Without loss of generality, we pick $x_{j}$ as the last node, which give a tree rooted at $x_{j}$. This operation makes the nodes in $T\left[x_{j}\right]$ be "promoted" up to the root node, which is shown in Fig. 2.

Assume node $x_{k}$, one of the ancestors of $T\left[x_{j}\right]$, is the neighboring node of $x_{j}$. Then $T\left[x_{k}\right]$ is consisted of the nodes, $\left\{x_{i} \mid x_{i} \notin\right.$ $\left.T\left[x_{j}\right]\right\}$, not in $T\left[x_{j}\right]$. According to Lemma 1, the sub-graph of $G$

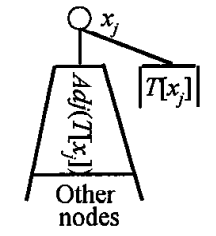

Fig. 2 The elimination tree structure after composite rotations consisting of nodes not in $T\left[x_{j}\right]$ is connected too.

Theorem 3. For any $k<n_{g}$, the Algorithm 1 could guarantee that the rest part of the graph $G$ is connected after all the sub-graphs $\left\{C_{j}, j=1,2, \ldots, k\right\}$ have been eliminated from $G$.

Proof. For any $k<n_{g}$, without loss of generality, based on Algorithm 1 and Lemma 1 we assume that $C_{i} \cup C_{i+1} \cup \cdots \cup C_{k}$ $(i<k)=T\left[x_{k}\right]$, in which $x_{k}$ is the last node of MD ordering in $C_{k}$, while $C_{l}, C_{2}, \ldots, C_{i-1}$ are consisted of disjoint subtrees respectively. Since there is no interaction between any pair of the disjoint subtrees, Lemma 2 could be iteratively used for these disjoint subtrees. Therefore the rest part of the graph $G$ is still connected after eliminating all the disjoint subtrees $C_{l}, C_{2}, \ldots, C_{t-l}, T\left[x_{k}\right]$, which means the rest part of the graph $G$ is connected after all the sub-graphs $\left\{C_{j}, j=1\right.$, $2, \ldots, k\}$ have been eliminated from $G$.

MD reordering still has another favorable property for frontier minimization of the proposed graph partitioning algorithm. Consider a node $x_{j}$ in the MD reordering $x_{1}, x_{2}$, $\ldots, x_{n}$. Let $C_{j}$ be the connected component in the sub-graph $\left\{x_{l}, \ldots, x_{j}\right\}$ that contains the node $x_{j}$. If we use $\operatorname{Adj}_{G}\left(C_{j}\right)$ to denote the adjacent set of the node subset $C_{j}$, then the property could be expressed as follows:

Theorem 4. [16] The adjacent set \{degree\} of the node $x_{j}$ at elimination is given by $\operatorname{Adj}_{G}\left(C_{j}\right)\left\{\left|\operatorname{Adj}_{G}\left(C_{j}\right)\right|\right\}$.

Theorem 4 assures that the MD reordering based multiway graph partitioning algorithm could provide a minimum frontier node set $\left|\operatorname{Adj}_{G}\left(C_{j}\right)\right|$ for any sub-graph $C_{j}$, which is crucial for getting the final optimal partition. This is largely due to the very nature of the MD ordering scheme. At step $j$ of the ordering algorithm, a node $x_{j}$ is selected with the minimum degree $\left|A d j_{G}\left(C_{j}\right)\right|$. The local minimization of the degree in the elimination graph has the desirable effect of controlling the size of the adjacent set $\operatorname{Adj}_{G}\left(C_{j}\right)$, and hence the size of the corresponding frontier node set.

Hence, the weighted MD reordering based multi-way graph partitioning algorithm, which has a sound theoretical basis, guarantees that any power network can be partitioned into desired number of connected sub-networks with quasibalanced FSE burdens and local minimum frontier elements.

\section{B. Frontier reduction algorithm}

After performing the Algorithm 1, the frontier node sets crossing different sub-graphs are determined as well, which will be further minimized to reduce the interaction of adjacent sub-graphs. Joseph has suggested a node separator improving method ${ }^{[14]}$, which is applied to minimize the number of the node separators in 2-way graph partitioning. We extend the node separator improving method to perform the frontier reduction in multi-way graph partitioning.

Let $\operatorname{Adj}_{G}(x, U)$ or $A d j(x, U)$ denote the adjacent nodes of $x$ in $U$, that is, $\operatorname{Adj}(x, U)=A d j(x) \cap U$. The same way, for a subset $W, \operatorname{Adj}(W, U)=\operatorname{Adj}(W) \cap U$. For any obtained subgraph $C_{i}\left(i=1,2, \ldots, n_{g}\right)$, the initial value of its corresponding frontier node set is represented by $F_{i}(i=1,2$, $\left.\ldots, n_{g}\right)$ and defined as:

$$
F_{i}=\left\{u \mid u \in \operatorname{Adj}\left(C_{i}, U\right), U=\bigcup_{j>i} C_{j}, i, j=1,2, \ldots, n_{g}\right\}
$$

Consider a subset $Y$ of any given frontier node set $F_{i}$, where $\operatorname{Adj}\left(Y, C_{i}\right) \neq C_{i}$. The following simple but crucial results are stated.

Proposition 5: Suppose $F_{i}$ is the frontier node set crossing $C_{i}$ and $U$, then the set $\bar{F}_{i}=\left(F_{i}-Y\right) \cup \operatorname{Adj}\left(Y, C_{i}\right)$ is the frontier node set of the two sets $\bar{C}_{i}=C_{r}-\operatorname{Adj}\left(Y, C_{l}\right)$ and $\bar{U}=U \cup Y$. Proposition 6: If $\left|\operatorname{Adj}\left(Y, C_{i}\right)\right|<|Y|$, then $\left|\bar{F}_{i}\right|<\left|F_{i}\right|$.

It can be observed that the central issue of the frontier reduction algorithm is how to determine a subset $Y$ of any frontier node set $F_{i}$ so that the size of $\operatorname{Adj}\left(Y, C_{i}\right)$ is less than that of $Y$. This problem is much associated with a wellknown combinatorial problem called bipartite graph matching ${ }^{[14,17]}$. All the necessary material, bipartite graph, matching, saturated or unsaturated vertex, alternating path, augmenting path and other related concepts can be found in [14] and [17]. Then the frontier reduction algorithm is described systematically in Algorithm 2. 
Step I: $i=1$

Algorithm 2. Frontier reduction algorithm

Step 2: For the sub-graph $C_{i}$ and its frontier node set $F_{i}$, construct the corresponding bipartite $H_{1}$ by $\left(F_{1}, \operatorname{Adj}_{G}\left(F_{1}, C_{1}\right), E\right)$. At the same time, initialize matching $M=\varnothing$.

Step 3. For each node $x \in F_{i}$, generating the $M$-alternating tree rooted at the node $x$ which is defined as: $L_{0}, L_{l}, L_{2}, \ldots, L_{2 j-1}, L_{2 j}, \ldots$, where:

$L_{0}=\left\{x_{0}\right\} ; j=1,2,3, \ldots ;$

For odd levels: $I_{2, j}=A d j_{H}\left(L_{0} \cup \cdots \cup L_{2 j-2}\right)$;

For even levels: $L_{2 j}=\left\{u \mid\{u, v\} \in M\right.$ for some $\left.u \in L_{3_{-l}}\right\}$

If an $M$-augmenting path starting from $x$ is found, then augment the current matching $M$ by this path; repeatedly perform Step 3 until all nodes of $F_{i}$ have been tested, then go to Step 4. else

according to the alternating tree rooted at $x$, return the desired $Y$ by the union of all the even levels, including $L_{0}$. That is,

$Y=L_{0} \cup L_{2} \cup \cdots \cup L_{2}$

get the modified $\bar{C}$ and $\bar{F}$ based on the Proposition 5 .

break and restart this algorithm from Step 2.

Step 4: $i=i+1$, if $i=n_{g}$, then this frontier reduction algorithm terminates, else go to Step 2.

We still use the simple power network in Fig. 1 as the illustrative example, which has been redrawn in Fig. 3. On

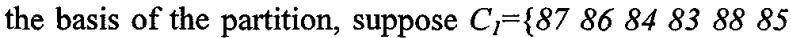
$90918982\}$ and then its corresponding frontier node set is

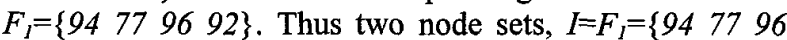
92\}, $J=\operatorname{Adj}_{G}\left(F_{l}, C_{l}\right)=\{828991\}$, and the edge set $E$ crossing $I$ and $J$ construct a bipartite graph $H=(I, J, E)$. If $M=\{\{77,82\},\{96,89\},\{92,91\}\}$ is the matching of the bipartite graph, it can be seen that node 94 is a $M$ unsaturated node in $I$, so we could get the alternating tree rooted at node 94 by (4): $L_{0}=\{94\}, L_{1}=\{82\}, L_{2}=\{77\}$, and thus the expected subset of the frontier node set $F_{1}$ is $Y=\{94, \quad 77\}$, which has a smaller adjacent set $\operatorname{Adj}_{H}(Y)=\{82\}$.

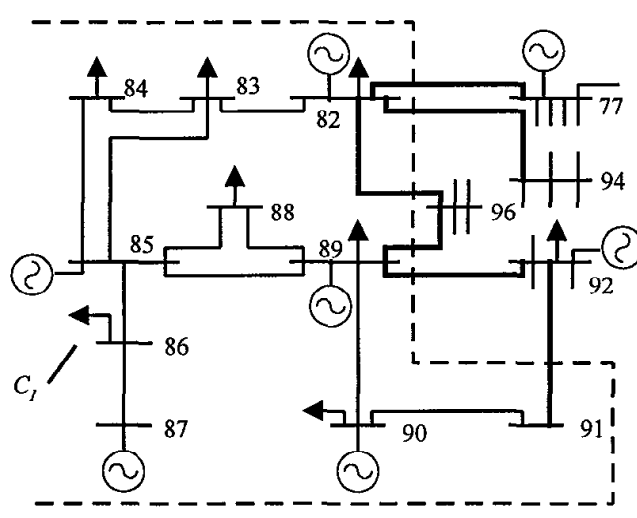

Fig. 3 The illustrative power network for frontier reduction algorithm

In a word, for any large-scale power network, the two steps of the multi-way graph partitioning method described in this section are performed in sequence. Consequently the optimal graph partitioning solution for FSE could be obtained systematically.

\section{COMPUTER SIMULATION RESULTS}

The proposed multi-way graph partitioning method has been implemented with sparse storage technique. The sparse storage technique only stores and operates nonzero elements and this could improve the calculation efficiency greatly.
IEEE 14-bus, 30-bus and 118-bus systems are used as the test systems respectively. The case, IEEE 118-bus system and $n_{g}=12$, is selected as an example to show the working process of the proposed multi-way graph partitioning method systematically. After forming the weighted MD ordering, Algorithm $I$ is performed to get $n_{g}$ $=12$ sub-networks. The corresponding initial value of the sub-networks and frontier node sets are listed in Table 1. In addition, the initial frontier and the reduction results of the Algorithm 2 are displayed in Table 2. However, it only reduces the frontier node sets by one frontier node, which is mainly due to the simplicity of the test system.

From this case, it can be concluded that the proposed multiple-way graph partitioning method works effectively and could satisfy all the requirements of FSE problem simultaneously. Other cases in IEEE 14-bus, 30-bus and 118-bus systems could get the similar results. We list these results in Table 3. In this table, we will not display every element in sub-network sets and frontier node sets. On the contrast, because the expected weights of each subnetwork, denoted by aver_wt, can be calculated by aver_w $w=$ (the total weights of the whole network) $/ n_{g}$, we use $\max \left\{\mid w t\left(C_{t}\right)-\right.$ aver_wt $\left.\mid\right\} / a v e r_{\text {_wt }}$ to represent the unbalance degree of the partition. The smaller the unbalance degree, the more balanced the obtained subnetworks.

Table 1. Obtained sub-networks of IEEE 118-bus system after partition

\begin{tabular}{|c|l|c|}
\hline$i=\left[1, n_{g}\right]$ & \multicolumn{1}{|c|}{ Obtained sub-networks $C_{F}=\left\{x \mid x \in C_{l}\right\}$} & $w l\left(C_{l}\right)$ \\
\hline 1 & $1,2,3,4,5,6,7,8,9,10$ & 25 \\
\hline 2 & $50,51,52,53,54,55,56,57,58$ & 25 \\
\hline 3 & $82,83,84,85,86,87,88,89,90,91$ & 25 \\
\hline 4 & $103,104,105,106,107,108,109,110,111,112$ & 25 \\
\hline 5 & $92,93,94,95,96,97,101,102$ & 22 \\
\hline 6 & $24,70,71,72,73,74,75,76,118$ & 24 \\
\hline 7 & $25,26,27,28,29,31,32,113,114,115$ & 26 \\
\hline 8 & $11,12,13,14,15,16,17,18,33,117$ & 25 \\
\hline 9 & $19,20,21,22,23,34,35,36,43,44$ & 22 \\
\hline 10 & $59,60,61,62,63,64,66,67$ & 22 \\
\hline 11 & $30,37,38,39,40,41,42,45,46,47,48,49$ & 30 \\
\hline 12 & $65,68,69,77,78,79,80,81,93,99,100,116$ & 26 \\
\hline
\end{tabular}

Table 2. The frontier node sets of IEEE 118-bus system

\begin{tabular}{|c|l|l|}
\hline$i=\left[1, n_{g}-1\right]$ & $\mathrm{F}_{i}$ before reduction & $F_{i}$ after reduction \\
\hline 1 & $11,12,30$ & No change \\
\hline 2 & 49,59 & No change \\
\hline 3 & $77,92,96$ & 92,82 \\
\hline 4 & 100 & No change \\
\hline 5 & 80,100 & No change \\
\hline 6 & $23,69,77$ & No change \\
\hline 7 & $17,23,30$ & No change \\
\hline 8 & $19,30,37$ & No change \\
\hline 9 & 37,45 & No change \\
\hline 10 & 49,65 & No change \\
\hline 11 & 65,69 & No change \\
\hline
\end{tabular}

Table 3. The test results of IEEE 14-bus, 30-bus and 118-bus systems

\begin{tabular}{|l|c|c|c|c|c|c|}
\hline & \multirow{2}{*}{$\begin{array}{c}\text { 14-bus } \\
\left(n_{g}=2\right)\end{array}$} & \multicolumn{2}{|c|}{30 -bus } & \multicolumn{3}{|c|}{ 118-bus } \\
\cline { 3 - 7 } & $n_{g}=2$ & $n_{g}=3$ & $n_{g}=2$ & $n_{g}=3$ & $n_{g}=8$ \\
\hline $\begin{array}{l}\text { Unbalance } \\
\text { degree \% }\end{array}$ & 11.7 & 12.6 & 31 & 19.2 & 12.1 & 13.1 \\
\hline $\begin{array}{l}\text { Before } \\
\text { reduction (no.) }\end{array}$ & 2 & 3 & 4 & 3 & 7 & 20 \\
\hline $\begin{array}{l}\text { After } \\
\text { reduction (no.) }\end{array}$ & 2 & 3 & 4 & 3 & 7 & 20 \\
\hline
\end{tabular}

The results in Table 3 have demonstrated that the proposed method could not only work effectively but also succeed in all the test power networks. In addition, it 
should be pointed out that the reduction effect of Algorithm 2 is not obvious for the above test cases, which is caused by the simplicity of the test systems.

For comparison purpose the graph partitioning method based on depth-first-search (DFS) algorithm ${ }^{[17]}$ has been realized and tested as well. Compared with the proposed graph partitioning method, though the DFS based graph partitioning method can satisfy all the requirements of FSE, it can not effectively minimize the number of the frontier nodes since DFS tree has no effect on minimization of the frontiers. Table 4 compares the number of the frontier nodes between these two methods. The results show that the proposed multiple-way graph partitioning method has better quality than DFS based graph partitioning method.

Table 4. The comparison between MD reordering and DFS based method
\begin{tabular}{|c|c|c|c|c|c|c|}
\hline & $\begin{array}{c}14-\text {-bus } \\
\left(n_{g}=2\right)\end{array}$ & $n_{g}=2$ & $n_{g}=3$ & $n_{g}=2$ & $n_{g}=3$ & $n_{g}=8$ \\
\hline $\begin{array}{c}\text { No. of } F_{1} \\
\text { based on DFS }\end{array}$ & 2 & 3 & 5 & 5 & 10 & 21 \\
\hline $\begin{array}{c}\text { No. of } F_{1} \\
\text { based on MD }\end{array}$ & 2 & 3 & 4 & 3 & 7 & 20 \\
\hline
\end{tabular}

\section{CONCLUSIONS}

In this paper an efficient $\mathrm{MD}$ reordering based graph partitioning method for distributed fault section estimation system in large-scale power networks is proposed. The partitioning procedure and characteristic analysis is presented. The analysis demonstrates that the proposed method can effectively satisfy all the requirements of the FSE. The method has been implemented with sparse storage technique and tested in the IEEE 14-bus, 30-bus and 118bus systems. For comparison purpose the graph partitioning method based on DFS algorithm has been realized and tested as well. Computer test results show that the proposed multiple-way graph partitioning approach is suitable for distributed fault section estimation system in large-scale power networks and has better quality as compared with the graph partitioning method based on DFS algorithm.

\section{ACKNOWLEDGMENTS}

The research is supported by National Key Basic Research Special Fund (No. G1998020305), P.R. China, to whom sincere acknowledgement are expressed.

\section{REFERENCES}

[1] C. Fukui and J. Kawakami, "An expert system for fault section estimation using information from protective relays and circuit breakers", IEEE Trans. Power Delivery, Vol. 1, No. 4, October 1986, pp. 83-90.

[2] M. Ernesto Vazquez, L. Oscar, M. Chacon, J. Hector and F. Altuve, "An on line expert system for fault section diagnosis in power system", IEEE Irans. Power Systems, Vol.12, No.1, February 1997, pp.357-362.

[3] Hyun-Joon Cho and Jong Keun Park, "An expert system for fault section diagnosis of power systems using fuzzy relations", IEEE Trans. Power Systems, Vol.12, No.1, February 1997, pp.342-348.

[4] V. Navarro, A.L. da Silva, L.A.V. de Carvalho and G. Zaverucha, "Artificial neural networks for power systems diagnosis", 1994 IEEE International Conference on Neural Networks, 1994, pp. 37383743.

[5] Z.E. Aygen, S. Seker, M. Bagriyanik, F.G. Bagriyanik and E. Ayaz, "Fault section estimation in electrical power systems using artificial neural network approach", 1999 IEEE Transmission and Distribution Conference, 1999, pp. 466-469.

[6] C.S. Chang, L. Tian and F.S. Wen, "A new approach to fault section estimation in power systems using ant system", Electric Power System Research, 1999(41), pp. 63-70

[7] T.S. Sidhu, O. Cruder and G.J. Huff, " An abductive inference technique for fault diagnosis in electrical power transmission networks", IEEE Trans. Power Delivery, Vol.12, No.1, January 1997, pp.515-522.

[8] M.R Garey and D.S Johnson, "Computer and interact-ability: a guide to the theory of NP-completeness", W.H. Freeman\&Co., 1979.

[9] B W Kernighan and S. Lin, "An efficient heuristic procedure for partitioning graphs", Bell Syst. Tech. J., Vol.49, February 1970, pp. 291-307.

[10] C.H. Lee, C.I. Park and M. Kim, "Efficient algorithm for graphpartitioning problem using a problem transformation method", Computer-Aided Dess., Vol. 21, No.10, 1989, pp.611-618.

[11] F. Qian, H. Hirata, "A parallel learning cellular automata for combinatorial optimization problems", Proceedings of IEEE International Conference on Evolutionary Computation, 1996, pp. 553-558.

[12] L. Tao and Y.C. Zhao, "Effective heuristic algorithms for VLSI circuit partition", IEE Proceedings-G: Circuits, Devices and Systems, Vol. 140, No. 2, April 1993, pp.127-134.

[13] J.R. Gilbert and E. Zmijewski, "A parallel graph partitioning algorithm for a message passing mutiprocessor", Int'l. J. Parallel Programming, Vol. 16, 1987, pp. 427-449.

[14] Joseph W.H. Liu, "A graph partitioning algorithm by node separators", ACM Trans. On Mathematical Software, Vol.15, No.3, September 1989, pp. 198-219.

[15] D.J. Rose, "A graph-theoretic study of the numerical solution of sparse positive definite systems of linear equations", In Graph Theory and Computing, R. Read, Ed. Academic Press, Orlando, Fla., 1972, pp. 183-217.

[16] Joseph W.H. Liu, "The role of elimination trees in sparse factorization", SIAM J. MATRIX ANAL. APPL., Vol. 11, No. 1, January 1990, pp. 134-172.

[17] T.S. Bi, Y.X. Ni, C.M. Shen and F.F. Wu, "An Efficient Graph Partition Method for Fault Section Estimation in Large-Scale Power Network", Proceedings of IEEE power engineering society winter meeting, 2001.

\section{BIOGRAPHIES}

Tianshu Bi received her B. Eng, and M. Eng degrees from North China Electric Power University, P. R. China, in 1994 and 1997 respectively. She is currently a Ph. D. candidature at the Dept. of E.E.E., the University of Hong Kong. Her research area includes power system operation, protective relay and the application of artificial intelligence in power systems.

Yixin Ni received her B. Eng., M. Eng., and Dr. Eng. from Electrical Engineering Department, Tsinghua University, P. R. China in 1968, 1981 and 1983 respectively. Her research interest is in power system modeling, simulation, stability and control, and power electronics applications in power systems. She was a professor of Tsinghua University and is now with the University of Hong Kong.

C.M. Shen received his B.Sc. (Eng.) and M.Sc. (Eng.) degrees in EE from the University of Hong Kong (HKU) in 1963 and 1965 respectively and his Ph.D. degree in EE from Queen Mary College, University of London in 1969. Since then he has joined the HKU. His research interest is in Load flow, fault analysis, state estimation and stability and control of power systems. He is an executive committee member of the Specialized Section in Power, IEE Hong Kong Centre.

Felix F. Wu joined the University of Hong Kong as Chair professur of Electrical Engineering in September 1995 and is now Pro-vice-chancellor of HKU. Prior to that, he was Professor and Vice Chairman, Department of Electrical Engineering and Computer Sciences, University of California, Berkeley, the same institute where he received his Ph.D. degree. His research interests are in power system planning and operation, including economics and reliability in system planning, real-time security assessment, and design of energy management systems and distribution automation. Recently he has been involved in the design of industry restructuring and electricity pricing. 